\title{
ON LAME'S DIFFERENTIAL EQUATION AND ELLIPSOIDAL HARMONICS
}

\author{
By E. T. Whittaker. \\ [Received and Read December 10th, 914.]
}

\section{Introductory.}

The object of the present paper is in the first place to establish the following theorem :-

The solutions of the homogeneous integral equation

$$
y(x)=\lambda \int_{0}^{4 \kappa} P_{n}(k \operatorname{sn} x \operatorname{sn} s) y(s) d s
$$

(where $n$ is a positive integer, $P_{n}$ is Legendre's function, and $k$ is the modulus, and $4 K$ the period of the elliptic functions $\mathrm{sn}$ ) are solutions of Lamé's differential equation

$$
\frac{d^{2} y}{d x^{2}}=\left\{n(n+1) k^{2} \mathbf{s n}^{2} x+A ; y .\right.
$$

and, in fact, are precisely those solutions of Lamé's differential equation which are rational in sn $x$.

It is well known that the differential equation (2) has no solutions which are rational in $\operatorname{sn} x$, except when the constant $A$ has one of a certain sequence of values - the "Eigenwerte" or " characteristic values" or "auto-values" of $A$. The integral equation (1) does not involve $A$ at all, but it involves the constant $\lambda$, which does not occur in the differential equation, and the integral equation (1) has no solution (other than zero) except when $\lambda$ has one of a certain sequence of Eigenwerte. The theorem asserts that the solutions of (1) corresponding to the Eigenwerte of $\lambda$ are the same as the solutions of (2) corresponding to the Eigenwerte of $A$.

This theorem is applied in the later part of the paper in order to solve 
a problem which has been present to my mind for some years pastnamely, that of expressing the "ellipsoidal harmonies" (that is to say, the solution of Laplace's equation

$$
\frac{\hat{\sigma}^{2} V}{\hat{r} x^{2}}+\frac{\partial^{2} V}{\partial y^{2}}+\frac{\partial^{2} V}{\partial z^{2}}=0
$$

which are appropriate to the ellipsoid), in the form which, as I showed in 1902,* represents the general solution of Laplace's equation, namely,

$$
V=\int_{0}^{2 \pi} f(x \cos \theta+y \sin \theta+i z, \theta) d \theta
$$

It may be recalled that most of the well-known particular solutions of Laplace's equation can be readily expressed in this form; for instance, the fundamental spherical harmonic, cylindrical harmonic, and ellipticcylindrical harmonic solutions can be expressed respectively by the equations

$$
\begin{aligned}
& r^{n} P_{n}^{n}(\cos \theta) \cos m \phi=\text { constant } \times \int_{0}^{2 \pi}(x \cos \theta+y \sin \theta+i z)^{n} \cos m \theta d \theta \\
& e^{-k x} J_{n n}(k \rho) \cos m \phi=\text { constant } \times \int_{0}^{2 \pi} e^{i k(x \cos \theta+y \sin \theta+i z)} \cos m \theta d \theta \\
& e^{-k z} c e_{n}(\xi) c e_{n}(i \eta)=\text { constant } \times \int_{0}^{2 \pi} e^{i n(x \cos \theta+y \sin \theta+i z)} c e_{n}(\theta) d \theta
\end{aligned}
$$

But the ellipsoidal harmonies, which are products of Lame's functions, resisted all attempts to express them in this form ; and, indeed, I satisfied myself that it was impossible to obtain such an expression of them by use of any of the properties of Lamés functions then known. The discovery of the integral equation (1), however, has now enabled me to show (in $\$ 4$ ) that any ellipsoidal harmonic in the rectangular coordinates $(x, y, z)$ can be expressed in the form

$$
\int_{0}^{4 K} P_{n} \frac{1}{k^{\prime} c}\left(k^{\prime} x \operatorname{sn} s+y \text { en } s+i z d n s\right) l_{j} E(s) d s,
$$

where $P_{n}$ denotes Legendre's function, and $E(s)$ denotes a Lame's function. This expression leads (in $\S 5$ ) to a very general property possessed 
by all Lamés functions, which can be expressed by the equation

$$
\begin{aligned}
& E(\alpha) E(\beta) E(\gamma) \\
& =\lambda \int_{0}^{4 \kappa} P_{n}\left(k^{2} \operatorname{sn} \alpha \operatorname{sn} \beta \operatorname{sn} \gamma \operatorname{sn} s-\frac{k^{2}}{k^{2}} \operatorname{cn} \alpha \operatorname{cn} \beta \operatorname{cn} \gamma \operatorname{cn} s\right. \\
& \left.-\frac{1}{k^{\prime 2}} \operatorname{dn} \alpha \operatorname{dn} \beta \operatorname{dn} \gamma \operatorname{dn} s\right) E(s) d s,
\end{aligned}
$$

where $\alpha, \beta$, and $\gamma$ are any three quantities. By assigning special constant values to $\beta$ and $\gamma$, an infinite number of integral equations for Lamés functions can be obtained, of which that obtained in the beginning of the present paper is one.

2. Derivation of the Fundamental Integral Equation.

We shall now proceed to establish the result numbered (1) above.

For this purpose we shall require a property possessed by the Legendrian function $P_{n}(k \operatorname{sn} x$ sn $s)$, namely, that it is a solution of the partial differential equation

$$
\frac{\partial^{2} u}{\partial x^{2}}-\frac{\partial^{2} u}{\partial s^{2}}=n(n+1) k^{2}\left(\operatorname{sn}^{2} x-\operatorname{sn}^{2} s\right) u .
$$

To prove this, we have by direct differentiation,

$$
\begin{aligned}
& \frac{\partial^{2}}{\partial x^{2}} P_{n}(k \operatorname{sn} x \operatorname{sn} s)-\frac{\partial^{2}}{\partial s^{2}} P_{n}(k \operatorname{sn} x \operatorname{sn} s) \\
= & P_{n}^{\prime \prime}(k \operatorname{sn} x \operatorname{sn} s) k^{2}\left(\mathrm{cn}^{2} x \mathrm{dn}^{2} x \mathrm{sn}^{2} s-\mathrm{cn}^{2} s \mathrm{dn}^{2} s \mathrm{sn}^{2} x\right) \\
& \quad+2 k^{3} P_{n}^{\prime}(k \operatorname{sn} x \operatorname{sn} s) \operatorname{sn} x \operatorname{sn} s\left(\mathrm{sn}^{2} x-\mathrm{sn}^{2} s\right) \\
= & k^{2}\left(\mathrm{sn}^{2} x-\operatorname{sn}^{2} s\right)\left[\left(k^{2} \mathrm{sn}^{2} x \mathrm{sn}^{2} s-1\right) P_{n}^{\prime}(k \operatorname{sn} x \sin s)\right. \\
& \left.\quad+2 k \operatorname{sn} x \operatorname{sn} s P_{n}^{\prime}(k \operatorname{sn} x \operatorname{sn} s)\right] \\
= & n(n+1) k^{2}\left(\operatorname{sn}^{2} x-\operatorname{sn}^{2} s\right) P_{n}(k \operatorname{sn} x \operatorname{sn} s),
\end{aligned}
$$

which establishes the result.

Now, let $I(x)$ denote the integral

$$
\int_{0}^{4 K} P_{n}(k \operatorname{sn} x \operatorname{sn} s) y(s) d s,
$$


1914.] Lamé's differential eqUation aNd ELLIPSOIDal hakMonics. 263

where $y(s)$ denotes a solution of Lamés equation

$$
\frac{d^{2} y}{d s^{2}}=\left\{n(n+1) k^{2} \operatorname{sn}^{2} s+A ; y,\right.
$$

the solution in question being rational in sn $s$.

Then we have

$$
\begin{aligned}
& \frac{d^{2} I}{d x^{2}}-\left\{n(n+1) k^{2} \operatorname{sn}^{2} x+A ! I\right. \\
&= \int_{0}^{4 K}\left[\frac{\partial^{2}}{\partial x^{2}} P_{n}(k \operatorname{sn} x \operatorname{sn} s)-n(n+1) k^{2} \operatorname{sn}^{2} x P_{n}(k \operatorname{sn} x \operatorname{sn} s)-A P_{n}\left(k \operatorname{sn} x \operatorname{sn}^{\prime} s\right)\right] \\
& \times y(s) d s .
\end{aligned}
$$

Using the above property (5) of the Legendrian function, the right-hand side of this equation becomes

$$
\begin{array}{r}
\int_{0}^{4 K}\left[\frac{\partial^{2}}{\partial s^{2}} P_{n}(k \text { sn } x \text { sn } s)-n(n+1) k^{2} \operatorname{sn}^{2} s P_{n}(k \text { sn } x \text { sn } s)-A P_{n}(k \text { sn } x \text { sn } s)\right] \\
\times !(s) d s .
\end{array}
$$

Substituting from equation (6), this becomes

$$
\begin{gathered}
\int_{0}^{4 K}\left[y(s) \frac{\partial^{2}}{\partial s^{2}} P_{n}(k \sin x \operatorname{sn} s)-P_{n}(k \operatorname{sn} x \operatorname{sn} s) \frac{d^{2} y}{d s^{2}}\right] d s, \\
{\left[y(s) \frac{\partial}{\partial s} P_{n}(k \sin x \operatorname{sn} s)-P_{n}(k \sin x \operatorname{sn} s) \frac{d y}{d s}\right]_{0}^{4 K},}
\end{gathered}
$$

and this is zero, since $P_{n}(k \operatorname{sn} x$ sn $s)$ and $y(s)$ have $4 K$ as a period.

The integral $I$ is therefore a solution of the equation

$$
\frac{d^{2} y}{d x^{2}}-\left\{n(n+1) k^{2} \operatorname{sn}^{2} x+A ; y=0 ;\right.
$$

and from its form it is evidently a rational function of sn $x$. But there cannot be more than one independent solution of the equation (7) which is rational in sn $x$ : and this is the solution which, when $s$ was taken as variable, was denoted by $y(s)$. Therefore $I$ must be a constant multiple of this solution $y(x)$; that is to say, the solutions of Lamé's equation (7) which are rational in sin $x$ satisfy the homogeneous integral equation

$$
y(x)=\lambda \int_{0}^{t R} P_{n}(k \operatorname{sn} x \text { sn } s) y(s) d s .
$$




\section{A Particular Case.}

As an illustration we shall verify this result for one of the simplest of Lamé's functions, namely,

$$
E(x)=k^{2} \mathbf{s n}^{2} x-t,
$$

where

$$
t=\frac{1}{3}\left\{1+k^{2}+\sqrt{ }\left(1-k^{2}+k^{4}\right)\right\} .
$$

As we shall find, the verification by direct integration is by no means obvious, but requires the recurrence formulæe between the integrals of elliptic functions, as well as the algebraic properties of $t$.

For this particular function we have $n=2$, so the theorem to be verified is

$$
E(x)=\lambda \int_{0}^{4 K}\left(3 k^{2} \operatorname{sn}^{2} x \operatorname{sn}^{2} s-1\right)\left(k^{2} \operatorname{sn}^{2} s-t\right) d s .
$$

Now we have $\frac{d}{d s}(\operatorname{sn} s \operatorname{cn} s \mathrm{dn} s)=1-2\left(1+k^{2}\right) \operatorname{sn}^{2} s+3 k^{2} \cdot \operatorname{sn}^{4} s$.

Integrating with respect to $s$ between the limits 0 and $4 K$, we see that if the integrals $\int_{0}^{4 K} d s$ and $\int_{0}^{4 K} \mathrm{sn}^{2} s d s$ are denoted by $I$ and $J$ respectively, we have

$$
\int_{0}^{4 K} \operatorname{sn}^{4} s d s=\frac{1}{3 k^{2}}\left\{2\left(1+k^{2}\right) J-I\right\}
$$

Substituting in equation (8), the integral on the right hand becomes

or

$$
\begin{gathered}
t I-k^{2}\left(1+3 t \mathrm{sn}^{2} x\right) J+k^{2} \mathrm{sn}^{2} x\left\{2\left(1+k^{2}\right) J-I\right\}, \\
-I\left(k^{2} \operatorname{sn}^{2} x-t\right)-k^{2} J\left\{1+3 t \operatorname{sn}^{2} x-2\left(1+k^{2}\right) \mathrm{sn}^{2} x\right\} .
\end{gathered}
$$

But since $t$ satisfies the equation

we have

$$
3 t^{2}-2 t\left(1+k^{2}\right)+k^{2}=0,
$$

$$
3 t-2\left(1+k^{2}\right)=-\frac{k^{2}}{t} ;
$$

and therefore the expression may be written

or

$$
\begin{gathered}
-I\left(k^{2} \mathrm{sn}^{2} x-t\right)-\frac{k^{2} J}{t}\left(k^{2} \sin ^{2} x-t\right), \\
-\left(I+\frac{k^{2} J}{t}\right) E(x),
\end{gathered}
$$

which establishes the result. 
1914.] Lame's differentiat. equation and eLLIPSOIdal harmonics. 265

\section{Expression of the General Ellipsoidal Harmonic.}

We shall now obtain an expression for the general ellipsoidal harmonic in the form

$$
\int_{0}^{2 \pi} f(x \cos \theta+y \sin \theta+i z, \theta) d \theta .
$$

Let

$$
\frac{x^{2}}{\lambda^{2}}+\frac{y^{2}}{\lambda^{2}-b^{2}}+\frac{z^{2}}{\lambda^{2}-c^{2}}=1
$$

be a family of confocal quadrics. The ellipsoidal harmonics associated with this family are defined to be the product of one of the quantities

$$
1, x, y, z, \quad y z, z x, \quad x y, \quad x y z,
$$

by an expression of the type

$$
\begin{aligned}
\left(\frac{x^{2}}{t_{1}}+\frac{y^{2}}{t_{1}-b^{2}}+\frac{z^{2}}{t_{1}-c^{2}}-1\right)\left(\frac{x^{2}}{t_{2}}+\frac{y^{2}}{t_{2}-b^{2}}+\right. & \left.\frac{z^{2}}{t_{2}-c^{2}}-1\right) \\
& \ldots\left(\frac{x^{2}}{t_{k}}+\frac{y^{2}}{t_{k}-b^{2}}+\frac{z^{2}}{t_{k}-c^{2}}-1\right)
\end{aligned}
$$

(where $t_{1}, t_{2}, \ldots, t_{k}$ are constants), such that the whole product satisfies Laplace's equation

$$
\frac{\partial^{2} V}{\partial x^{2}}+\frac{\partial^{2} V}{\partial y^{2}}+\frac{\partial^{2} V}{\partial z^{2}}=0
$$

It is a well known theorem that there are always $(2 n+1)$ of these ellipsoidal harmonics of any degree $n$ in $(x, y, z)$. We shall denote any one of them by $G_{n}(x, y, z)$.

The terms of highest degree in $G_{n}(x, y, z)$ will evidently be

$$
\left\{\begin{aligned}
\left.\begin{array}{rll}
x & x & y z \\
y & z x & x y z
\end{array}\right\}\left(\frac{x^{2}}{t_{1}}+\frac{y^{2}}{t_{1}-b^{2}}+\frac{z^{2}}{t_{1}-c^{2}}\right)\left(\frac{x^{2}}{t_{2}}+\frac{y^{2}}{t_{2}-b^{2}}+\frac{z^{2}}{t_{2}-c^{2}}\right) \\
\cdots\left(\frac{x^{2}}{t_{k}}+\frac{y^{2}}{t_{k}-b^{2}}+\frac{z^{2}}{t_{k}-c^{2}}\right)
\end{aligned}\right.
$$

the large bracket indicating that some one of the quantities inside it is to be taken as a multiplier of the product outside. These terms of highest degree we shall denote by $H_{n}(x, y, z)$. It is obvious that $H_{n}(x, y, z)$ satisfies Laplace's equation on its own account; and being homogeneous of degree $n$ in $(x, y, z)$, it will be expressible in the form

$$
H_{n}(x, y, z)=\int_{0}^{2 \pi}(x \cos \theta+y \sin \theta+i z)^{n} f(\theta) d \theta,
$$

where $f(\theta)$ is not yet known. 
Now it is known* that

$$
G_{n}(x, y, z)=\left\{1-\frac{D^{2}}{2(2 n-1)}+\frac{D^{4}}{2.4(2 n-1)(2 n-3)}-\ldots\right\} H_{n}(x, y, z),
$$

where $D^{2}$ stands for $\quad a^{2} \frac{\partial^{2}}{\partial x^{2}}+\beta^{2} \frac{\partial^{2}}{\partial y^{2}}+\gamma^{2} \frac{\partial^{2}}{\partial z^{2}}$,

and $(\alpha, \beta, \gamma)$ are the semi-axes of any one of the quadrics of the family.

Substituting for $H_{n}(x, y, z)$ the above value, we have

$$
G_{n}(x, y, z)
$$$$
=\int_{0}^{2 \pi}\left\{A^{n}-\frac{n(n-1)}{2(2 n-1)} A^{n-2} B^{2}+\frac{n(n-1)(n-2)(n-3)}{2.4(2 n-1)(2 n-3)} A^{n-4} B^{4}-\ldots\right\} f(\theta) d \theta \text {, }
$$

where $A=x \cos \theta+y \sin \theta+i z$,

and

$$
B=\sqrt{ }\left(\alpha^{2} \cos ^{2} \theta+\beta^{2} \sin ^{2} \theta-\gamma^{2}\right)=\sqrt{ }\left(c^{2}-b^{2} \sin ^{2} \theta\right),
$$

so

$$
G_{n}(x, y, z)=\int_{0}^{2 \pi} P_{n}\left(\frac{A}{B}\right) f(\theta) d \theta
$$

where $P_{n}$ denotes Legendre's function ; a constant factor and a $B^{n}$ having been absorbed into the $f(\theta)$.

Thus we have the ellipsoidal harmonic expressed in the form

$$
G_{n}(x, y, z)=\int_{0}^{2 \pi} P_{n}\left(\frac{x \cos \theta+y \sin \theta+i z}{\sqrt{\left(c^{2}-b^{2} \sin ^{2} \theta\right)}}\right) f(\theta) d \theta,
$$

where $f(\theta)$ is a function as yet undetermined.

The form of this result suggests a change of variable. Let $s$ be a new variable introduced in place of $\theta$, and defined by the equation

$$
\sin \theta=\frac{\mathrm{cn} s}{\operatorname{dn} s},
$$

where the elliptic functions are formed with the modulus $k=b / c$. Then we have

$$
\frac{\cos \theta}{\sqrt{\left(c^{2}-b^{2} \sin ^{2} \theta\right)}}=\frac{\operatorname{sn} s}{c}, \quad \frac{\sin \theta}{\sqrt{\left(c^{2}-b^{2} \sin ^{2} \theta\right)}}=\frac{\operatorname{cn} s}{k^{\prime} c}, \quad d \theta=-\frac{k^{\prime} d s}{\operatorname{dn} s},
$$

and the formula for $G^{n}(x, y, z)$ becomes

$$
G_{n}(x, y, z)=\int_{0}^{4 K} P_{n}\left\{\frac{1}{k^{\prime} c}\left(k^{\prime} x \operatorname{sn} s+y \operatorname{en} s+i z \operatorname{dn} s\right)\right\} \phi(s) d s .
$$

where $\phi(s)$ is a function which we shall now proceed to determine. 
In place of $(x, y, z)$ introduce coordinates $(\alpha, \beta, \gamma)$ defined by the equations

$$
\begin{aligned}
& x=c k^{2} \operatorname{sn} \alpha \operatorname{sn} \beta \text { sn } \gamma, \\
& y=-c \frac{k^{2}}{k^{\prime}} \text { en } \alpha \text { cn } \beta \text { en } \gamma, \\
& z=\frac{i c}{k^{\prime}} \operatorname{dn} \alpha \operatorname{dn} \beta \operatorname{dn} \gamma,
\end{aligned}
$$

the elliptic functions being formed with the same modulus as before.

Then the equations

$$
a=\text { constant }, \quad \beta=\text { constant }, \quad \gamma=\text { constant, }
$$

represent respectively the three families of surfaces of the confocal family : and it is known from the way in which ellipsoidal harmonics are built up of Lamé's functions that the ellipsoidal harmonic $G_{n}(x, y, z)$ considered either as a function of $a$ alone, or as a function of $\beta$ alone, or as a function of $\gamma$ alone, satisfies Lamé's differential equation.

Now $G$ in its new form is

$$
\begin{aligned}
G_{n}(x, y, z)=\int_{0}^{4 K^{\circ}} P_{n}\left(k^{2} \operatorname{sn} \alpha \operatorname{sn} \beta \operatorname{sn} \gamma \operatorname{sn} s\right. & -\frac{k^{2}}{k^{\prime 2}} \operatorname{en} \alpha \operatorname{cn} \beta \text { en } \gamma \text { en } s \\
& \left.-\frac{1}{k^{\prime 2}} \operatorname{dn} \alpha \operatorname{dn} \beta \operatorname{dn} \gamma \operatorname{dn} s\right) \phi(s) d s,
\end{aligned}
$$

and this satisties Lamé's equation when regarded as a function of $\alpha$, whatever constant values may be assigned to $\beta$ and $\gamma$. Suppose then that we assign the value $K$ to $\beta$, and the value $K+i K^{\prime}$ to $\gamma$, so that

$$
\text { en } \beta=0, \quad \text { sn } \beta=1, \quad \operatorname{dn} \gamma=0, \quad \text { sn } \gamma=\frac{1}{k} \text {. }
$$

Thus

$$
\int_{0}^{4 k} P_{n}(k \operatorname{sn} a \operatorname{sn} s) \phi(s) d s
$$

is a solution of Lame's equation in $a$. Comparing this with the result of $\$ 2$, we see that

$$
\phi(s)=E(s),
$$

where $E(s)$ is a Lame's function, and indeed is the particular Lamés function associated with the ellipsoidal harmonic considered. Thus substituting this value of $\phi(s)$ in equation (9), we have the required expression for a general ellipsoidal harmonic, namely,

$G_{n}(x, y, z)=$ constant $\times \int_{0}^{2 \pi} P_{n}\left\{\frac{1}{\bar{k}^{\prime} c}\left(k^{\prime} x\right.\right.$ sn $s+y$ en $\left.\left.s+i z \operatorname{dn} s\right)\right\} E(s) d s$. 
268 LaMé's differential equation and FLLipgotdal harmonics.

5. A General Property of Lamé's Functions.

If in equation (10) we express the ellipsoidal harmonic as a product of three Lame's functions, the equation becomes

$E(\alpha) E(\beta) E(\gamma)$

$=$ constant $\times \int_{0}^{4 K} P_{n}\left(k^{2} \operatorname{sn} a \operatorname{sn} \beta\right.$ sn $\gamma$ sn $s-\frac{k^{2}}{k^{\prime 2}}$ en $a$ en $\beta$ en $\gamma$ en $s$

$$
\left.-\frac{1}{k^{\prime 2}} \operatorname{dn} \alpha \operatorname{dn} \beta \operatorname{dn} \gamma \operatorname{dn} s\right) E(s) d s .
$$

If in this equation (which includes as a particular case the integral equation obtained in $\S 2$ ) we regard any two of the quantities $\alpha, \beta, \gamma$-say $\beta$ and $\gamma$-as constants, we obtain a homogeneous integral equation for the Lamé's function $E(a)$, with a symmetrical nucleus. It is obvious that an infinite number of homogeneous integral equations satisfied by the Lame's function $E(\alpha)$ can be obtained by assigning special values to $\beta$ and $\gamma$, or differentiating, \&c., with respect to one of them. 